\title{
Application of the Alpha Shape Method to Visualize and Analyze Surgical Motion
}

\author{
Mohammad R. Maddah, Caroline G. L. Cao \\ Department of Biomedical, Industrial, and Human Factors Engineering, Wright State University, Dayton, OH, USA \\ Email: maddah.2@wright.edu, caroline.cao@wright.edu
}

How to cite this paper: Maddah, M.R. and Cao, C.G.L. (2017) Application of the Alpha Shape Method to Visualize and Analyze Surgical Motion. Surgical Science, 8, 464-480.

https://doi.org/10.4236/ss.2017.811052

Received: June 30, 2017

Accepted: November 14, 2017

Published: November 17, 2017

Copyright (๑) 2017 by authors and Scientific Research Publishing Inc. This work is licensed under the Creative Commons Attribution International License (CC BY 4.0).

http://creativecommons.org/licenses/by/4.0/

(c) (i) Open Access

\begin{abstract}
Simulators play an important role in training surgery residents to perform laparoscopy surgery. Some of these simulators have the capability to track tool motion to assess performance. However, most have not utilized the data to analyze trainee performance in a meaningful way. The alpha shape method can be used to construct a geometric surface based on motion data to enable visualization of the performance, while the surface derivative (surface/time to completion)-efficiency-can be used as a metric to evaluate complex surgical performance. The utility of the alpha shape method was demonstrated in a pick-and-place task, where the motion path of laparoscopic graspers was recorded by a position sensor, miniBIRD $500^{\circledR}$. An alpha shape method was used to measure the surface area of the $3 \mathrm{D}$ points in space occupied by the tool tips during task performance. Results show that the surface derivative measure alone may be able to model the speed-accuracy tradeoff function, thereby simplifying the analysis and evaluation of complex motion in surgical performance.
\end{abstract}

\section{Keywords}

Surface, Alpha Shape, Motion Visualization, Performance Assessment

\section{Introduction}

Traditional open surgery in the abdominal cavity has largely been replaced with the modern approach of laparoscopic or minimally invasive surgery (MIS) [1]. In MIS, the space within the abdomen is insufflated with carbon dioxide to increase the working volume. Operations are performed through small incisions in the abdominal wall, typically between $0.5-1.5 \mathrm{~cm}$. Trocars are placed through these incisions to allow introduction of surgical instruments (endoscopic camera, graspers, scissors, etc.) into the surgical field. The MIS approach, compared 
with the open approach in surgery, is more demanding physically and cognitively for the surgeon, and requires a great deal more training before practicing on patients [2]. The most significant advantages of laparoscopic surgery are shortened recovery time, less bleeding, and reduction in post-operative pain and suffering for the patient. As patient safety in laparoscopic surgery is heavily dependent on the surgeon's training and experience with laparoscopic tools [3], the need for effective training, especially for surgical residents and novices to this surgical approach, has ignited tremendous new interest in the surgical education community surrounding the acquisition and assessment of surgical skills, and a unifying metric to measure performance.

The commonly used simulators for training laparoscopic surgical skills are box trainers (e.g., SAGES FLS [4]), and computer-based virtual reality systems (e.g., LapSim). These training systems are designed to record trainee performance data, such as time, errors, and, in some, 3D position of surgical tools. Based on these measures, learning and performance can be assessed by various performance measures.

Motor learning science has shown that expert-level skill performance is characterized by high speed and accuracy in execution, as well as smoothness of movement [5]. The degree of task automaticity, or how much the task can be performed without pre-planning and focused attention, is another indication of expertise [6]. For simple motor skills, the performance metric is simply defined by the measured reduction in these performance parameters (i.e., speed, accuracy, smoothness of motion, etc.) For more complex tasks, such as surgery, the relative importance of the various performance parameters can change depending on what the task is, which surgical procedure is being performed, or even on the condition of the patient, resulting in a complex metric that must be based on task requirements [7], as well as functional and clinical definitions of goodness of performance.

In addition to speed (time to task completion) and accuracy (error), several researchers have proposed using motion-related measures to assess surgical skill performance [8] [9] [10] [11]. Surgical tool path length and deviation, economy of movement, idle states, depth, total and partial time, idle time, speed, acceleration, motion smoothness, and force interactions can be measured and used in varying combinations to assess performance in a specific surgical skill. For example, "time" and "number of hand movements" has been shown to correlate with levels of expertise, while the measured path length has not [8] [10]. Researchers have shown that surgical tool "motion smoothness" and "idle time" during surgery correlated highly with surgical tasks requiring bimanual coordination [11].

Even though "number of movements" and "motion smoothness", as measures of skill acquisition and performance, offer a better representation of the multivariate construct of skill performance, the hallmark of expert performanceefficiency and consistency-has been more difficult to derive from motion 
tracking data. The purpose of this paper is to present a new methodology for visualizing and analyzing motion tracking data to assess performance-the alpha surface reconstruction technique. Alpha surface reconstruction is an algorithm typically used for creating a surface from unorganized clouds of $2 \mathrm{D}$ or $3 \mathrm{D}$ points. It uses Delaunay triangulation to form a surface called "alpha shape". The alpha shape surface can be refined depending on the desired granularity for surface representation. We posit that this alpha shape method can be used alone, or in conjunction with the other performance measures such as time and accuracy, to measure the efficiency and consistency of performance by trainees while executing a surgical task. By tracking the 3D location of the laparoscopic tool end-effectors in a box trainer, surgical trainees' tool manipulation can be visualized as a 3D geometrical surface with a specific size.

\section{Alpha Surface Construction}

In the field of geometrical representation and data visualization, there are many methods available to construct a surface from a set of unorganized 3D points. Most of the existing algorithms have been used for surface reconstruction of an object in the real world. The data in these methods are usually acquired from 3D scanners, cameras, or from mathematical models [12]. The most commonly used surface reconstruction algorithms are based on the interpolation (B-Spline) method [13], the least square method [14], Poisson surface reconstruction [15], and Voronoi/Delaunay triangulation [12] [16]. Selection of the technique for implementation is based on its strength in dealing with specific features of the object being reconstructed. Traditionally, the B-Spline method is used for a curve or surface, and volume fitting over a set of points in two and three dimensions. A spline is a piecewise polynomial function which covers all the space to form a curve/surface in two or three dimensions [17] [18]. The least square method is another fitting method in which errors are calculated based on the differences between points on the reconstructed surface and the given data. The obtained errors are iteratively minimized to achieve the greatest similarity between the real object and constructed model [19]. For analysis of laparoscopic skill performance in a box trainer, the least square and B-Spline methods are thus not suitable. They do not allow the identification and removal of unwanted points (i.e., the points that are not related surgical task performance, see section 2.3) or noise from the data set before initiating surface reconstruction.

The Poisson method is a method used for reconstructing surfaces based on 3D data points. In this method, a set of $3 \mathrm{D}$ points, $\mathrm{V}$, are used to find a gradient surface. The points are to be fitted on the gradient surface $\chi$ which is obtained by solving the Poisson equation: $\nabla \cdot \nabla \chi=\nabla \cdot \mathrm{V}[15]$. The Poisson algorithm uses all the given points at the same time to generate a surface, hence it is known as a global solution for surface reconstruction problems. Poisson surface reconstruction produces a smooth surface over all the given points, filling in all the holes (low density areas) in the dataset. In the case of the surgical tool motion in our 
laparoscopic training box, the created Poisson surfacewould result in a much larger surface than the actual surface, as the isolated low-density areas are also connected to the created surface from the task space proper. Therefore, the Poisson method is not appropriate for our purpose.

The Delaunay triangulation (DT) method has been shown to be efficient and accurate for dense and noise-free data [12]. It generates a mesh of triangles which can cover most of the points [12]. DT algorithms check for the "empty circle" test: a circle (sphere if in 3D) crossing any 3 points in the data must not include any other point in the enclosed space. Figure 1 shows the accepted and rejected triangulation for four sample points $\{\mathrm{A}, \mathrm{B}, \mathrm{C}$, and $\mathrm{D}\}$.

The Delaunay triangulation method does not control for the size of the triangles. It produces larger triangles in sparsely populated regions in which points are far from one another and smaller triangles in dense regions in which points are very close to one another. Hence it takes into account all unwanted points for surface reconstruction. This could cause the reconstructed surface to resemble a larger convex hull with a larger measured area than the actual object (Figure 2).

Alpha shape algorithm is a method derived from Delaunay triangulation (DT) methods [20] [21]. The major difference between DT and the alpha shape algorithm is the additional constraint in the latter-the radius of each circle passing through the three vertices of the triangles must be less than a predefined value, alpha $(\alpha)$ (Figure 1). The alpha value ranges from zero to infinity, and generates different shapes, called alpha shape, depending on the alpha value. For $\alpha=0$, the alpha shape is the point itself. When $\alpha$ is chosen large enough (infinity), the alpha shape is a convex hull which is the output of the DT algorithm. The alpha value can be determined experimentally to achieve a balance between size and shape/surface contours. Alternatively, a pre-determined alpha value can be set to allow the comparison of sizes and shape/surface of reconstructed data sets.

The concept of alpha shapes can be generalized to data points in three dimensions. According to the formal definition in Edelsbrunner's earlier work [22], for a given set of 3D points $S$, an $\alpha$-ball was defined as a sphere with the radius of $\alpha$.
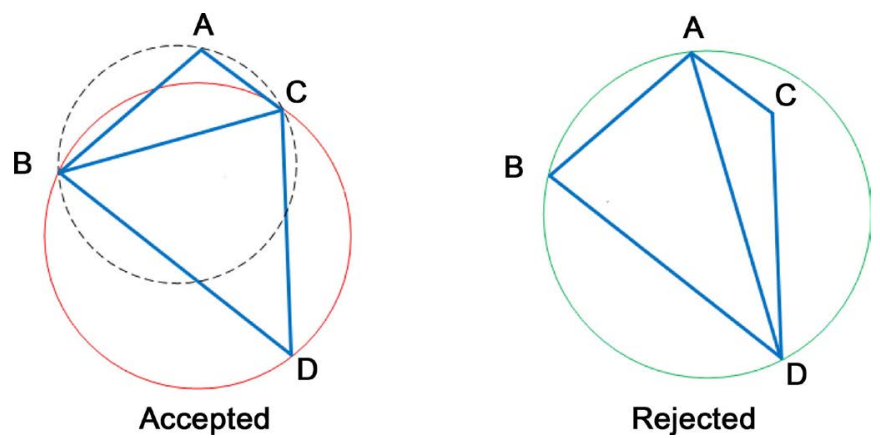

Figure 1. Empty circle test for Delaunay triangulation. Accepted triangulation: A and D do not lie in the interior of the circles passing through "BCD" (red circle) and "ABC" (black-dashed circle), respectively. Rejected triangulation: $\mathrm{C}$ lies in the interior of the circle passing through "ABD” (green circle). 


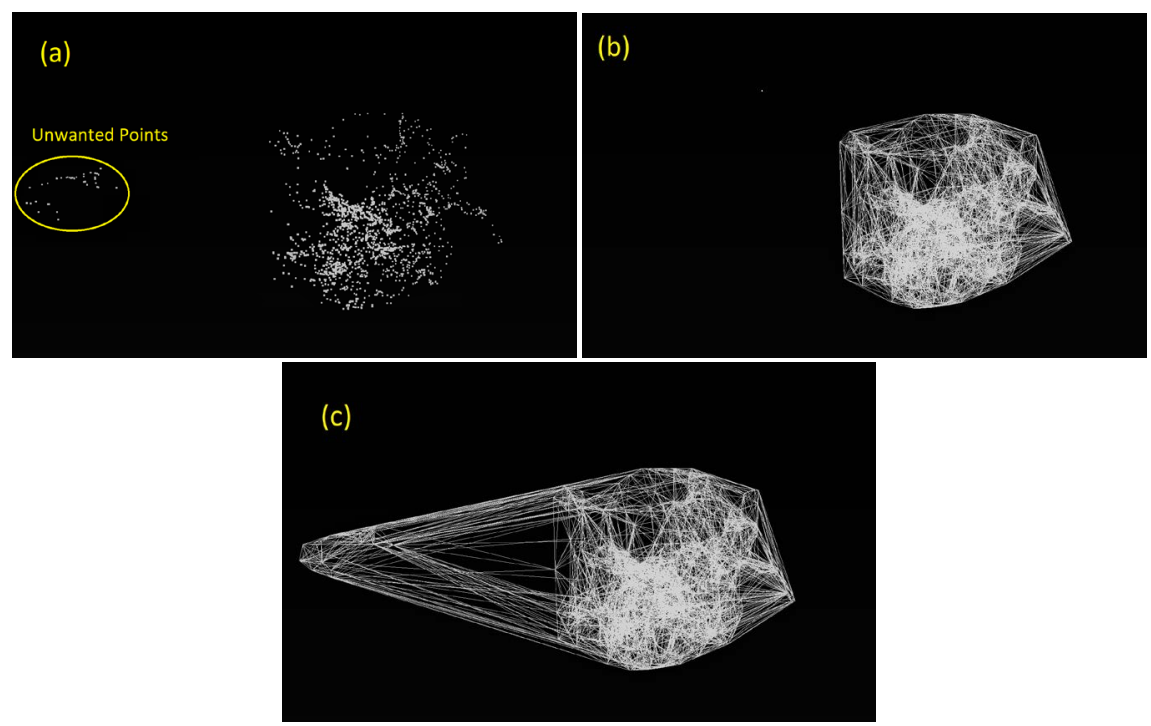

Figure 2. Delaunay triangulation (a) input points (unwanted data points are indicated by the yellow circle on the left) (b) convex hull with extraneous points removed (c) convex hull with extraneous points included.

The $\alpha$-ball, $b$, moves around the entire space $S$ and their intersection $T=b \cap S$ can then include a number of points ranging between a single data point $(\alpha=0)$ and all the data points $(b=S)$. Any subset $T$ of $S(T \subseteq S)$ with the size of $\mathrm{k}+1$, $0 \leq \mathrm{k} \leq 3$, define a k-simplex which is a convex hull of the points in $T$. For the larger size of the $T$ inside the $\alpha$-ball, the convex hull (obtained from Delaney triangulation and consisting of $\mathrm{k}$-simplexes with $0 \leq \mathrm{k} \leq 2$ ) is called an $\alpha$-exposed shape $F_{k, \alpha}$. In fact, inside any $\alpha$-ball in the $S$, the alpha shape is a polytope in which $F_{2, \alpha}$ triangles are its boundary, $F_{1, \alpha}$ lines are the edges and $F_{0, \alpha}$ points are the vertices of the triangles. The $\alpha$ parameter can play a role in controlling the surface size which makes it an attractive feature for data analysis. It can be used to remove the noisy and irrelevant data points from the dataset (see section 2.5). The alpha shape method, thus, appears to be the superior technique for the analysis of motion-related performance data in laparoscopic surgery.

To evaluate the alpha shape method, we analyzed data collected from an experiment that was conducted to examine the value of training technical and non-technical skills simultaneously in laparoscopic surgery using a box trainer [23]. The hypothesis was that training both technical and non-technical skills at the same time would be more efficient for learning the new skill. Time to task completion and error results from that experiment were compared with motion efficiency and consistency results from the alpha shape method. The experimental design is described in brief again here.

\section{Methods}

\subsection{Subjects}

Twenty-six participants were divided into three different groups according to their surgical experience/training: Novices, Intermediates, and Experts. One 
subject from the Expert group was excluded from the study due to a malfunction of the data acquisition system during the experiment. The Novices were Wright State University engineering students who had no prior surgical experience. The Intermediate group consisted of surgical interns at the Miami Valley Hospital in Dayton, OH. These interns had each performed fewer than 50 laparoscopic training cases. Fourth- and fifth-year General Surgery and OB/GYN residents at the same hospital were considered Experts in our study as they had each performed more than 400 laparoscopic training cases. The study was approved by the Wright State University Institutional Review Board.

\subsection{Laparoscopic Training System}

A ProMIS simulator, with a torso-shaped laparoscopic training box, was used to simulate the laparoscopic surgery environment (Figure 3). The trainer box was equipped with a built-in video camera located in the pubic bone area of the box, which provided a view of the workspace. Position trackers (Flock of Bird miniBirdsensors) were used to track the $3 \mathrm{D}$ position of the surgical tool tip at a sampling rate of $12.82 \mathrm{~Hz}$. The workspace was displayed on a computer monitor that was placed beside the trainer box and clearly visible to the participants in the experiment. Inside the trainer box were placed a large paper cup $(7.1 \mathrm{~cm}$ diameter, $2.0 \mathrm{~cm}$ deep) containing small 3D objects, and two smaller paper cups (4.2 $\mathrm{cm}$ diameter, $2.4 \mathrm{~cm}$ deep). The large cup was positioned on one side (e.g., left) of the workspace inside the trainer box, while the two smaller cups were placed on the other side (e.g., right), as shown on the monitor (Figure 3).

The small 3D objects inside the large cup were made of balsam wood, forming geometric shapes similar to those employed by Shepard and Metzler (1971) in their classic mental rotation experiments [24]. These 3D objects were of two different shapes that were mirror images of each other (Figure 4).

\subsection{Task}

In the experiment, participants were asked to use laparoscopic graspers, inserted into the trainer box, to pick up the objects from the large cup and transfer them into either one of the smaller cups as quickly and as accurately as possible (without dropping the objects), without regard for the geometric orientation of

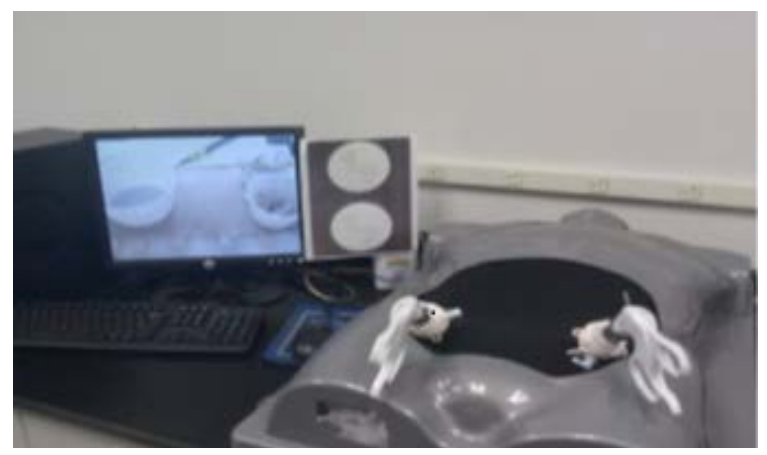

Figure 3. Torso-shaped laparoscopic training box (ProMIS simulator). 


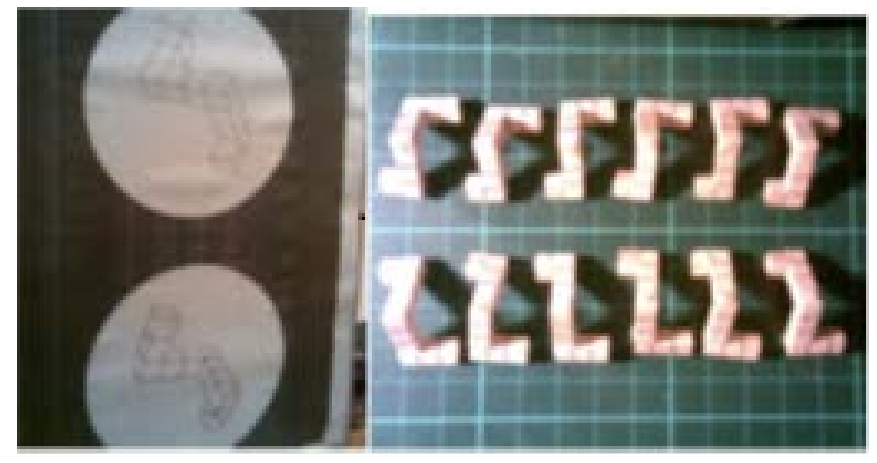

Figure 4. Two different geometrical shape of the objects. Shape laparoscopic training box (ProMIS simulator).

the objects. The pick-and-place task was a modified version of the FLS Peg Transfer task [4] and constituted the technical hand-eye coordination skill in basic laparoscopic surgery (training condition $=$ Technical). Alternatively, participants were asked to sort the objects into the two cups according to the orientation of the objects. A photo showing the classification of the two orientations was placed next to the monitor as a label for the object destinations (Figure 3 and Figure 4). In addition to the hand-eye coordination, required to perform the technical task, sorting required participants to perform mental rotation to classify the grasped object to one of two geometric orientations. Thus, the nontechnical skill was encapsulated in the mental rotation task (training condition = Combined). A third condition, to isolate the non-technical skill, required participants to sort the 3D objects as described, but using their hands to manipulate the objects directly, thus removing the technical component of the laparoscopic task (training condition $=$ Non-Technical). Once all the objects have been transferred, the cup positions were reversed and subjects had to repeat the transfer task in the opposite direction using the same graspers.

\subsection{Experimental Design}

Participants were divided evenly into one of three training groups with a different order of training (see Table 1).

Three subjects from each of the expertise levels were in each training group, resulting in 9 subjects per group. All participants performed one "pre-test" trial in the Combined condition, then 10 trials in each block of training (T, NT, C). At the end of each block, a test trial in the Combined condition was performed, with the last test trial being "post-test". Subjects in the Combined group were trained in blocks 2 and 3 only, so as to have equal exposure to the technical and non-technical aspects of the task as the other two groups. Performance measures such as time to task completion, errors (dropped objects and wrong sort), and tool tip 3D position were recorded throughout the trials.

\subsection{Motion Analysis-Alpha Shape Construction}

Performance measures such as "time to task completion" and "error" were ana- 
Table 1. Training program for an individual subject. Group $\mathrm{T}=$ Technical skill trials first, Group NT $=$ Non-Technical skill trials first, Group $\mathrm{C}=$ Combined skill trials first $\mathrm{T}=$ Technical, NT = Non-Technical, $\mathrm{C}=$ Combined.

\begin{tabular}{cccccccc}
\hline & Pre-test & $\begin{array}{c}\text { Training } \\
\text { Block 1 }\end{array}$ & test & $\begin{array}{c}\text { Training } \\
\text { Block 2 }\end{array}$ & test & $\begin{array}{c}\text { Training } \\
\text { Block 3 }\end{array}$ & Post-test \\
\hline Number of trials & 1 & 10 & 1 & 10 & 1 & 10 & 1 \\
Group T & $\mathrm{C}$ & $\mathrm{T}$ & $\mathrm{C}$ & $\mathrm{NT}$ & $\mathrm{C}$ & $\mathrm{C}$ & $\mathrm{C}$ \\
Group NT & $\mathrm{C}$ & $\mathrm{NT}$ & $\mathrm{C}$ & $\mathrm{T}$ & $\mathrm{C}$ & $\mathrm{C}$ & $\mathrm{C}$ \\
Group C & $\mathrm{C}$ & & & $\mathrm{C}$ & $\mathrm{C}$ & $\mathrm{C}$ & $\mathrm{C}$ \\
\hline
\end{tabular}

lyzed using pair-wise t-tests [25]. Post-test performance improvement over pretest performance would provide evidence to support learning; while the groupwise comparison results would support efficiency of training schedule (i.e., training technical skills first before non-technical skills, or vice versa, or combine the two skills in training).

The $3 \mathrm{D}$ positions of the laparoscopic tool tips were used to construct a surface of the tools' movements for each participant. The obtained surfaces reconstructed using the alpha shape algorithm would indicate the space that were swept by the surgical tools, and can be used as an index to differentiate the subjects' levels of expertise. That is, a larger swept area was expected for the novices, whereas smaller areas were expected for the experts.

Because the reconstructed shape is, in general, determined by the alpha value (radius of the alpha-ball), the best value of $\alpha$ in the alpha shape reconstruction algorithms is usually selected by trial-and-error and based on the final shape of the reconstructed object. For example, in a non-uniform distribution, the density varies locally and finding a global $\alpha$ which is perfectly suited for the entire space is impossible.Harada and $\mathrm{Xu}$ suggested a piecewise-linear technique in their alpha shape algorithm to choose dissimilar $\alpha$ values according to local densities [26]. As an alternative to finding the local densities, in this paper, local radii values were obtained from local Delaunay triangulations. The average value of the radii of the circles (spheres in 3D), which pass through vertices of the local Delaunay triangles, was selected as the global alpha value. As an example, $\alpha_{1}, \alpha_{2}$ and $\alpha_{3}$ are the local Delaunay radii for the given sampled points of "A", "B", " $\mathrm{C}$ ", " $D$ " and " $E$ " in Figure 5. The number of accepted triangles in the alpha algorithm is subject to the defined alpha value. For the biggest radius of $\alpha_{3}$, as a predefined alpha value, all the triangles in Figure 5 are accepted and for the smallest radius of $\alpha_{2}$, only "ABC" triangle is accepted. Furthermore, the median radius of $\alpha_{2}$ eliminates " $\mathrm{CDE}$ ", the biggest triangle, while accepting " $\mathrm{ABC}$ " and "BCD". Hence the median value is fit for the purpose of creating surfaces in dense regions such as "ABCD" in Figure 5. This method avoids unwanted points (e.g., "E") which are relatively far from the densest area. Therefore, the average of all local radii for each data set can be the alpha value which provides the maximum coverage of all the points while discarding all the unwanted points 


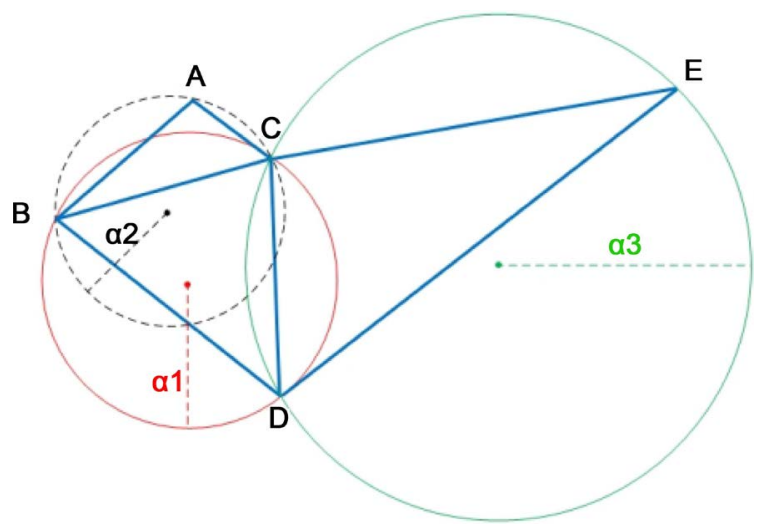

Figure 5. Two different geometrical shape of the objects. Shape laparoscopic training box (ProMIS simulator).

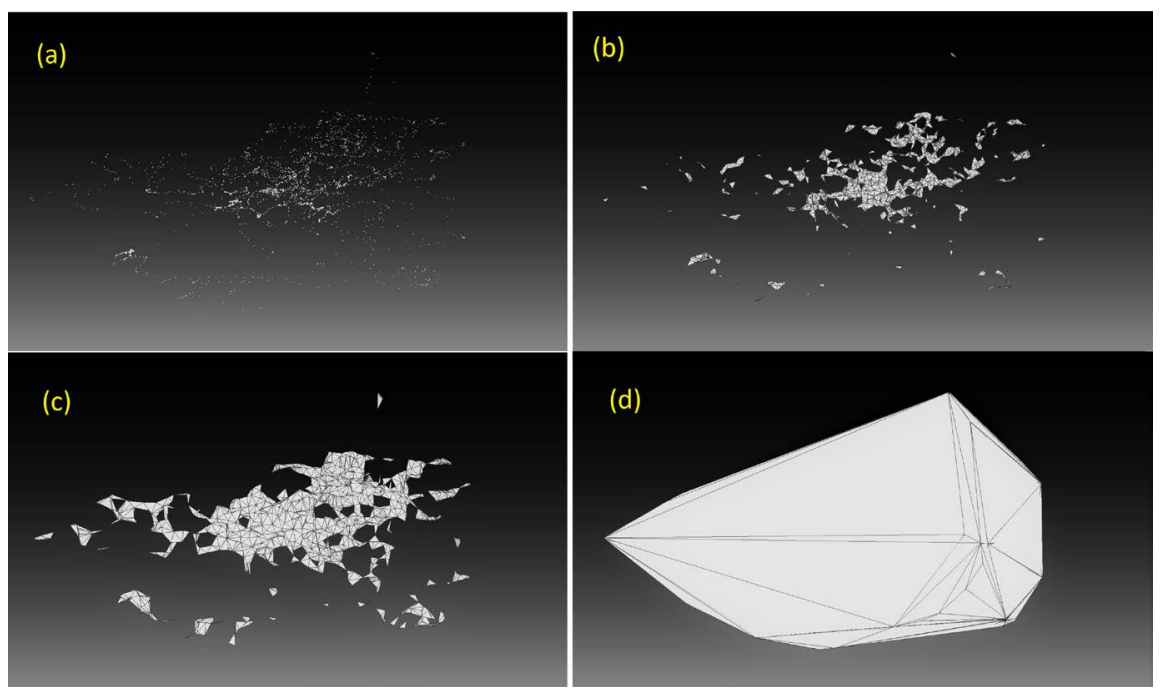

Figure 6. (a) 3D point cloud (input data for the alpha shape algorithm) from one subject during post-test; (b)-(d) different reconstructed alpha shapes for the input dataset with $\alpha$ $=0.06, \alpha=0.09$ and $\alpha=12.7$.

simultaneously.

Figure 6 shows the 3D point cloud of a subject in post-test, and the surfaces reconstructed using the alpha shape algorithm with $\alpha=0.06, \alpha=0.09$ and $\alpha=$ 12.7 for the same subject. Selected alpha value for this subject would be 0.067 which is the average value of local radii of Delaunay triangulation.

\section{Results}

The average radius of Delaunay triangle circles in pre- and post-test for experts, intermediates and novices were calculated and shown in Table 2. The calculated values in Table 2 represent the best $\alpha$ for each subject in each of the pre-test and post-test. For example, $\alpha=0.11$ was selected for pre-test surface of intermediate subject \#3, and $\alpha=0.09$ was selected for post-test surface of the same subject, as is shown in Figure 7. These alpha values were used to construct pre-test and post-test surfaces for individual participants. 
Table 2. Calculated alpha value for subjects in the expert, intermediate, and novice groups.

\begin{tabular}{ccccccc}
\hline \multirow{2}{*}{ Subject No. } & \multicolumn{2}{c}{ Novice Group } & \multicolumn{2}{c}{ Intermediate Group } & \multicolumn{2}{c}{ Expert Group } \\
\cline { 2 - 6 } & Pre-test & Post-test & Pre-test & Post-test & Pre-test & Post-test \\
\hline 1 & 0.13 & 0.20 & 0.04 & 0.08 & 0.06 & 0.10 \\
2 & 0.07 & 0.07 & 0.08 & 0.08 & 0.06 & 0.09 \\
3 & 0.04 & 0.07 & 0.11 & 0.09 & 0.07 & 0.10 \\
4 & 0.06 & 0.06 & 0.06 & 0.08 & - & - \\
5 & 0.05 & 0.07 & 0.05 & 0.08 & 0.06 & 0.11 \\
6 & 0.06 & 0.07 & 0.17 & 0.10 & 0.07 & 0.10 \\
7 & 0.06 & 0.07 & 0.05 & 0.08 & 0.07 & 0.06 \\
8 & 0.06 & 0.09 & 0.05 & 0.07 & 0.08 & 0.07 \\
9 & 0.08 & 0.13 & 0.09 & 0.09 & 0.08 & 0.10 \\
\hline
\end{tabular}
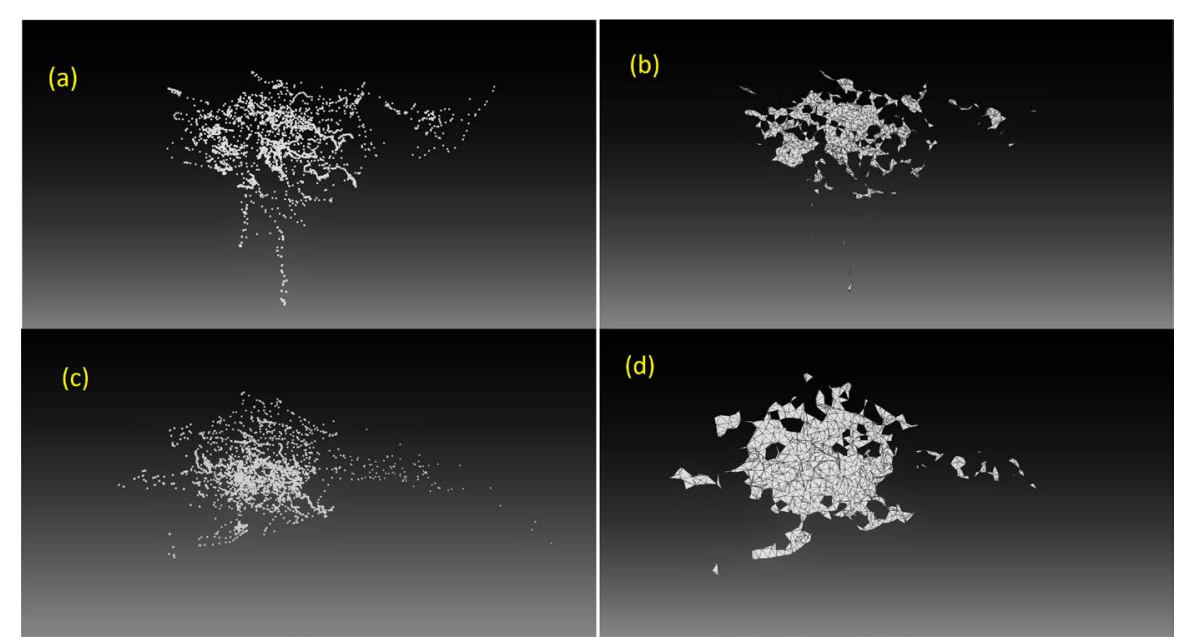

Figure 7. (a) Intermediate \# 3 pre-test $3 \mathrm{D}$ points (b) Intermediate\#3 pre-surface alpha $=$ 0.11 ; (c) Intermediate \# 3 post-test $3 \mathrm{D}$ points (b) Intermediate \# 3 post-surface alpha = 0.09 .

In addition to the calculated surfaces, connecting consecutive points of subjects' hand movements (see Figure 6(a)), the tool trajectory inside the training box can be measured by summing Euclidean distances from the start to the end point for each trial. The calculated average surfaces (using the alpha values in Table 2), trajectories, and "time to completion" for different training groups ( $\mathrm{T}$, NT and C) are shown in Table 3. Moreover, the averages of technical and nontechnical errors in Table 3 indicate the errors due to dropping and incorrect sorting of the objects [25].

Comparison of the average of reconstructed surfaces for pre-test and post-test in Table 3 shows that most of the post-test surface areas are smaller than pretest surfaces. To evaluate the interaction effects between the strategy and experience factors, and the contrast within the pre- and post-tests, a three factor ANOVA (test, experience and strategy) was performed on the results of surface, 
Table 3. Mean trajectory, surface, time, technical and non-technical errors of the expert, intermediate, and novice groups in different training strategies.

\begin{tabular}{cccccccccccc}
\hline & \multicolumn{2}{c}{ Mean Surface $(\mathrm{cm} 2)$} & \multicolumn{2}{c}{ Mean Time $(\mathrm{s})$} & \multicolumn{2}{c}{ Mean Trajectory $(\mathrm{cm})$} & \multicolumn{2}{c}{ Mean Tech $(\#)$} & \multicolumn{2}{c}{ Mean Non-Tech $(\#)$} \\
\cline { 2 - 11 } & Pre-test & Post-test & Pre-test & Post-test & Pre-test & Post-test & Pre-test & Post-test & Pre-test & Post-test \\
\hline Novice (T) & 25.30 & 18.33 & 153 & 92 & 157.69 & 99.82 & 0.33 & 0.67 & 1.3 & 0.3 \\
Novice (NT) & 28.89 & 21.78 & 186 & 95 & 163.21 & 114.3 & 3 & 0 & 1.7 & 0 \\
Novice (C) & 21.17 & 21.31 & 187 & 106 & 121.24 & 123.72 & 2.3 & 1 & 1.7 & 0 \\
Intermediate (T) & 32.58 & 31.96 & 160 & 112 & 346.6 & 151.56 & 2 & 1.3 & 1 & 0.33 \\
Intermediate (NT) & 42.18 & 28.26 & 252 & 113 & 564.4 & 155.43 & 2 & 0.67 & 2.3 & 0.33 \\
Intermediate (C) & 37.34 & 21.41 & 259 & 101 & 229.31 & 135.62 & 1.3 & 0 & 2 & 0.3 \\
Expert (T) & 28.81 & 26.47 & 137 & 99 & 176.4 & 179.86 & 0.67 & 1.3 & 1 & 0.67 \\
Expert (NT) & 25.89 & 17.08 & 130 & 160 & 194.19 & 114.63 & 1 & 1 & 1 & 0 \\
Expert (C) & 20.72 & 20.72 & 111 & 111 & 127.3 & 129.24 & 0 & 0 & 1.3 & 0.67 \\
\hline
\end{tabular}

trajectory, time, and technical and non-technical errors.

Surface. ANOVA results showed a significant effect of training on surfaces, $(F$ $(2,1)=25.44, p<0.001)$. There was also a significant main effect for experience $(F(2,1)=5.43, p=0.02)$ but no significant effect for strategy ( $\mathrm{T}, \mathrm{NT}$ and $\mathrm{C})$ $(F(2,1)=0.78, p=0.475)$. There was no significant interaction between strategy and experience $(F(2,1)=0.44, p=0.78)$. A post hoc Tukey HSD test indicated significant differences in overall surface between experts and intermediate participants $(p=0.042)$, and between novices and intermediate participants $(p=$ 0.023). Novices and experts were not different.

Time. A significant contrast between pre and post-test time to task completion was observed $(F(2,1)=44.285, p<0.001)$. There was a significant main effect for experience $(F(2,1)=4.969, p=0.02)$ whereas no difference was seen amongst training strategies $(F(2,1)=0.894, p=0.42)$. The interaction between strategy and experience was not significant $(F(2,1)=0.66, p=0.62)$.

Trajectory. There was a significant contrast between pre and post-test trajectories $(F(2,1)=5.156, p=0.036)$. Also, there was a significant main effect for experience $(F(2,1)=5.00, p=0.020)$ whereas no significant difference was observed within the training strategies $(F(2,1)=1.246, p=0.313)$. The interaction between strategy and experience was not significant $(F(2,1)=0.734, p=0.582)$.

Tech error. As reported in [25], both strategy $(F(2,1)=5.1, p=0.007)$ and experience level $(F(2,1)=7.7, p<0.001)$ showed a significant main effect in technical error. Also, a significant interaction between strategy and experience $(F(2,1)=8.04, p<0.001)$ was seen in technical error.

Non-Tech error. As reported in [25], there was no significant main effect for either strategy $(F(2,1)=0.47, p=0.63)$ or experience $(F(2,1)=2.3, p=0.10)$. There was, however, a significant interaction between the two factors $(F(2,1)=$ $8.69, p<0.001)$.

The surface measures from alpha shapes (the calculated areas of the alpha 
shapes) can be used to evaluate the efficiency of the subjects' performance. Efficiency is defined as the ratio of surface to time using the following equation:

Efficiency $=$ Surface (alpha shape area)/time to completion

The calculated efficiencies using the average surface and time from Table 3 are shown in Table 4. To evaluate any effects between the strategy-experience and the contrast within the pre-post average efficiencies, a three factor ANOVA (test, experience and strategy) was performed on the results in Table 4 for all the subjects. Results indicate a main significant contrast between pre- and post-test efficiencies $(F=26.19, p<0.001)$. Neither experience $(F(2,1)=1.40, p=0.27)$ nor strategy $(F(2,1)=1.38, p=0.28)$ showed a significant effect on efficiency.

Similar to the results for surface analysis, there was not any significant interaction between strategy and experience $(F=0.20, p=0.93)$.

To quantify the agreement between different performance measures, indices of time, trajectory, errors, and surface changes (deltas) were determined as follows:

$\Delta s=$ Pre-test(area)-Post-test(area)

$\Delta \mathrm{t}=$ Pre-test (time to completion)-Post-test (time to completion)

$\Delta t e=$ Pre-test (number of $\mathrm{T}$ errors)-Post-test (number of Terrors)

$\Delta$ nte $=$ Pre-test (number of NT errors)-Post-test (number of NT errors)

$\Delta \operatorname{tr}=$ Pre-test (trajectory length)-Post-test (trajectory length)

where $\Delta s, \Delta t, \Delta \operatorname{tr}, \Delta$ te and $\Delta$ nte represented improvements in surface, time, trajectory, "technical error" and "non-technical error", respectively (Table 5). For example, in the $\mathrm{T}$ training strategy (technical skill trials first), the average surface improvement delta was $6.97 \mathrm{~cm}^{2}$ for the novices, $0.63 \mathrm{~cm}^{2}$ for the intermediates and $2.34 \mathrm{~cm}^{2}$ for the experts (highlighted in Table 5). The similarity between surface improvement delta and the other performance measure deltas in Table 5 can be measured by mean correlation values (see Table 6).

Table 4. Mean efficiencies and technical errors of the expert, intermediate and novice groups in different training strategies. Groups: $\mathrm{T}=$ Technical, NT $=$ Non-Technical, $\mathrm{C}=$ Combined.

\begin{tabular}{ccccc}
\hline & \multicolumn{2}{c}{ Mean Efficiency $\left(\mathrm{cm}^{2} / \mathrm{s}\right)$} & \multicolumn{2}{c}{ Mean Technical Errors (\#) } \\
\cline { 2 - 5 } & Pre-test & Post-test & Pre-test & Post-test \\
\hline Novice (T) & 0.15 & 0.21 & 0.33 & 0.67 \\
Novice (NT) & 0.18 & 0.20 & 3 & 0 \\
Novice (C) & 0.13 & 0.19 & 2.3 & 1 \\
Intermediate (T) & 0.22 & 0.28 & 2 & 1.3 \\
Intermediate (NT) & 0.18 & 0.26 & 2 & 0.67 \\
Intermediate (C) & 0.15 & 0.21 & 1.3 & 0 \\
Expert (T) & 0.23 & 0.26 & 0.67 & 1.3 \\
Expert (NT) & 0.17 & 0.25 & 1 & 1 \\
Expert (C) & 0.19 & 0.20 & 0 & 0 \\
\hline
\end{tabular}


Table 5. The average of improvement deltas for different training schedules. Groups: $\mathrm{T}=$ Technical, NT = Non-Technical, $\mathrm{C}=$ Combined.

\begin{tabular}{|c|c|c|c|c|c|}
\hline & \multicolumn{5}{|c|}{ Novice } \\
\hline & $\Delta s\left(\mathrm{~cm}^{2}\right)$ & $\Delta \operatorname{tr}(\mathrm{cm})$ & $\Delta \mathrm{t}(\mathrm{s})$ & $\Delta$ te $(\#)$ & $\Delta$ nte $(\#)$ \\
\hline Group T & 6.97 & 57.87 & 61 & -0.34 & 1 \\
\hline Group NT & 7.11 & 48.91 & 91 & 3 & 1.7 \\
\hline \multirow[t]{3}{*}{ Group C } & -0.13 & -2.48 & 81 & 1.3 & 1.7 \\
\hline & \multicolumn{5}{|c|}{ Intermediate } \\
\hline & $\Delta s\left(\mathrm{~cm}^{2}\right)$ & $\Delta \operatorname{tr}(\mathrm{cm})$ & $\Delta \mathrm{t}(\mathrm{s})$ & $\Delta$ te $(\#)$ & $\Delta$ nte $(\#)$ \\
\hline Group T & 0.63 & 195.04 & 48 & 0.7 & 0.67 \\
\hline Group NT & 13.92 & 408.97 & 139 & 1.33 & 1.97 \\
\hline \multirow[t]{3}{*}{ Group C } & 15.93 & 93.69 & 158 & 1.3 & 0.7 \\
\hline & \multicolumn{5}{|c|}{ Expert } \\
\hline & $\Delta \mathrm{s}\left(\mathrm{cm}^{2}\right)$ & $\Delta \operatorname{tr}(\mathrm{cm})$ & $\Delta \mathrm{t}(\mathrm{s})$ & $\Delta$ te $(\#)$ & $\Delta$ nte $(\#)$ \\
\hline Group T & 2.34 & -3.46 & 38 & -0.63 & -0.67 \\
\hline Group NT & 8.81 & 79.56 & -30 & 0 & 1 \\
\hline Group C & 0.0 & -1.94 & 0 & 0 & 0.63 \\
\hline
\end{tabular}

Table 6. The cross correlations between improvement deltas for different training strategies.

\begin{tabular}{ccccc}
\hline Correlation & $\Delta \mathrm{t}(\mathrm{s})$ & $\Delta \operatorname{tr}(\mathrm{cm})$ & $\Delta$ te $(\#)$ & $\Delta$ nte $(\#)$ \\
\hline$\Delta \mathrm{s}\left(\mathrm{cm}^{2}\right)$ & 0.602 & 0.525 & 0.292 & 0.298 \\
\hline
\end{tabular}

Subscripts: $\mathrm{s}=$ surface, $\mathrm{t}=$ time, $\mathrm{tr}=$ trajectory, $\mathrm{te}=$ technical error, nte $=$ non-technical error.

The mean correlation value (regardless of training groups) between "surface" and "time" was higher than the other three measures.

\section{Discussion}

Depicting the tool movements in the simulated laparoscopic surgery task as point clouds allows for direct visualization of the subjects' performance. The calculated surface of the alpha shape from the point clouds provided a means for quantitative analysis of human performance.

The results of the ANOVA tests show that the surface data derived from the alpha method were able to differentiate between post- and pre-test performance and amongst expertise levels of the subjects (Table 3), similar to the "time to completion" and "trajectory" measures. Correlation results (Table 6) also confirm that the surface improvement deltas are strongly correlated with the time and trajectory deltas. These results suggest that the surface measure can be used as an index of learning or performance improvement, to the same extent that time and trajectory are measures of performance. Further analyses taking into account errors show that the surface measure is more than simply a surrogate of 
time or trajectory. Surface is poorly correlated with the number of technical errors committed by the subjects $(r=0.29)$ whereas time and trajectory are highly correlated with technical error $(r=0.52$ and 0.41 , respectively). This suggests that surface and technical error can be considered independent indices to evaluate subjects' motor performance.

We posit that efficiency, the derivative of surface measure (i.e., surface/time to completion), captures the speed-accuracy tradeoff phenomenon that is characteristic of skill performance during the learning stages. The speed-accuracy tradeoff phenomenon is observed when learners have not yet mastered the motor skill to the level of automaticity; speed is increased at the expense of higher error rates, while more accurate performance requires slowing down and taking more time to complete the task. Whereas speed (trajectory/time to completion) and error are both required to evaluate performance, efficiency alone is adequate to evaluate the performance of the subjects (Figure 8). Figure 8 illustrates the disparate measures of speed, accuracy, surface, and efficiency for the 3 expertise levels at baseline performance. Traditionally, speed and accuracy are taken together to subjectively evaluate the performer. That is, the expert performer is expected to be better (i.e., faster and/or more accurate) than the novice in task performance. If the observed speed is in fact lower, as in the case of the Intermediate versus Novice (Figure 8), one looks to the higher error measure to reconcile the discrepancy according to the speed-accuracy tradeoff function. A similar discrepancy is observed in the trajectory measure (Figure 8). The efficiency measure, on the other hand, fits the model of human performance with respect to expertise, and can provide a clear and concise score that takes into account the speed-accuracy function (Figure 8).

Efficiency, when applied to the analysis of skill performance in simulated laparoscopic surgery, was able to differentiate the subjects' expertise according to

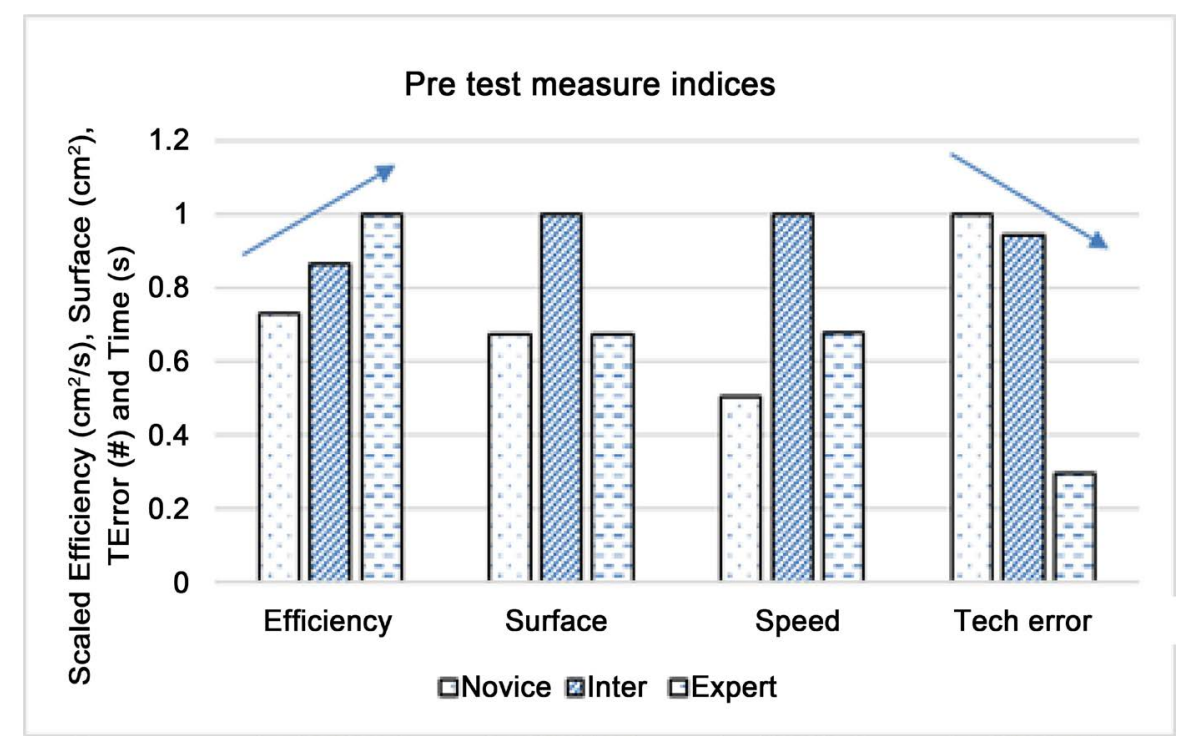

Figure 8. Pre-test measure indices: Speed, accuracy, surface and technical error vs. experience level. 
baseline performance, as well as measure the learning effect between pre-test and post-test.

In this paper, we assessed skill performance using surface, calculated from the alpha shape of point clouds, and compared the results with the measures of time, trajectory, technical and non-technical errors. Similar to the time and trajectory measures, the surface index can be used to partially assess performance. Moreover, we showed that surface and its derivative, efficiency of performance, can be an even more sensitive and complete indicator of performance as it takes into account the speed-accuracy tradeoff.

The role of alpha surface may be used in combination, or alone, to assess skill or learning, allowing the evaluation of consistency and efficiency of performance. In future, the concept of "surface" can be extended to a "manifold" in a higher dimension space to address other complex movements. The alpha surfaces can be replaced with topological manifolds in four-dimensional space in which every point has its coordinates as (x, y, z, vt) instead of (x,y, z). Thus, the alpha surface forms an alpha volume in 4-dimensional space and the time and surface assessment methods can be merged into a single time-space evaluation method.

\section{Acknowledgements}

This work was supported in part by the ONR SBIR Phase II award \# N0001410-M-0152, and the Ohio Third Frontier to the Ohio Imaging Research and Innovation Network (OIRAIN). The authors would like to acknowledge the following individuals for their assistance with data collection: Ting Sun, Subhashini Ganapathy, Amie Miller and Cristol Grosdemouge.

\section{References}

[1] Lee-Kong, S. and Daniel, L.F. (2013) The History of Minimally Invasive Surgery. Seminars in Colon and Rectal Surgery, 24, 3-6. https://doi.org/10.1053/j.scrs.2012.10.003

[2] Cao, C.G.L., MacKenzie, C.L. and Payandeh, S. (1996) Task and Motion Analyses in Endoscopic Surgery. Proceedings ASME Dynamic Systems and Control Division, 583-590.

[3] Rattner, D.W., Apelgren, K.N. and Eubanks, W.S. (2001) The Need for Training Opportunities in Advanced Laparoscopic Surgery. Surgical Endoscopy, 15, 1066-1070. https://doi.org/10.1007/s004640080021

[4] www.flsprogram.org/

[5] Fitts, P.M. (1964) Perceptual-Motor Skill Learning. Categories of Human Learning, 47, 381-391.

[6] Dreyfus, S.E. (2004) The Five-Stage Model of Adult Skill Acquisition. Bulletin of Science, Technology and Society, 24, 177-181. https://doi.org/10.1177/0270467604264992

[7] Gallagher, A.G. and Satava, R.M. (2002) Virtual Reality as a Metric for the Assessment of Laparoscopic Psychomotor Skills. Surgical Endoscopy and Other Interventional Techniques, 16, 1746-1752. https://doi.org/10.1007/s00464-001-8215-6 
[8] Datta, V., Mackay, S., Mandalia, M. and Darzi, A. (2001) The Use of Electromagnetic Motion Tracking Analysis to Objectively Measure Open Surgical Skill in the Laboratory-Based Model. Journal of the American College of Surgeons, 193, 479-485.

[9] Smith, C.D., Farrell, T.M., McNatt, S.S. and Metreveli, R.E. (2001) Assessing Laparoscopic Manipulative Skills. The American Journal of Surgery, 181, 547-550.

[10] Datta, V., Chang, A., Mackay, S. and Darzi, A. (2002) The Relationship between Motion Analysis and Surgical Technical Assessments. The American Journal of Surgery, 184, 70-73.

[11] Oropesa, I., Chmarra, M.K., et al. (2013) Relevance of Motion-Related Assessment Metrics in Laparoscopic Surgery. Surgical Innovation, 20, 299-312. https://doi.org/10.1177/1553350612459808

[12] Amenta, N., Bern, M. and Kamvysselis, M. (1998) A New Voronoi-Based Surface Reconstruction Algorithm. In: Proceedings of the 25th Annual Conference on Computer Graphics and Interactive Techniques, 415-421.

[13] Liu, X.M., Huang, H.K., Xu, W.X. and Chen, J. (2004) Research on the Reconstruction Method of B-Spline Surface Based on Radius Basis Function Neural Networks. In: Cybernetics and Intelligent Systems, Vol. 2, 1123-1127.

[14] Wen, P.Z., Wu, X.J., Zhu, Y. and Peng, X.W. (2009) LS-RBF Network Based 3D Surface Reconstruction Method. In: Chinese Control and Decision Conference, 5785-5789.

[15] Kazhdan, M. and Hoppe, H. (2013) Screened Poisson Surface Reconstruction. ACM Transactions on Graphics, 32, 29. https://doi.org/10.1145/2487228.2487237

[16] Amenta, N., Choi, S. and Kolluri, R.K. (2001) The Power Crust, Unions of Balls, and the Medial Axis Transform. Computational Geometry, 19, 127-153.

[17] Rogers, D.F. (2000) An Introduction to NURBS: With Historical Perspective. Elsevier.

[18] Bradley, C.H. and Vickers, G.W. (1993) Free-Form Surface Reconstruction for Machine Vision Rapid Prototyping. Optical Engineering, 32, 2191-2200. https://doi.org/10.1117/12.145064

[19] Ton, D. and Mayer, H. (2007) 3D Least-Squares-Based Surface Reconstruction. Photogrammetric Image Analysis PIA, 7.

[20] Bernardini, F., Bajaj, C.L., Chen, J. and Schikore, D.R. (1997) A Triangulation-Based Object Reconstruction Method. In: Proceedings of the Thirteenth Annual Symposium on Computational Geometry, 481-484. https://doi.org/10.1145/262839.263098

[21] Teichmann, M. and Capps, M. (1998) Surface Reconstruction with Anisotropic Density-Scaled Alpha Shapes. In: Proceeding IEEE Visualization, 67-72. https://doi.org/10.1109/VISUAL.1998.745286

[22] Edelsbrunner, H. and Mücke, E.P. (1994) Three-Dimensional Alpha Shapes. ACM Transactions on Graphics, 13, 43-72. https://doi.org/10.1145/174462.156635

[23] Grosdemouge, C., Weyhrauch, P., Niehaus, J., Schwaitzberg, S. and Cao, C.G.L. (2012) Design of Training Protocol for Perceptual and Technical Skills in a Minimally Invasive Surgery. In: ASME Biennial Conference on Engineering Systems Design and Analysis, 855-860. https://doi.org/10.1115/ESDA2012-82869

[24] Shepard, R. and Metzler, J. (1992) Mental Rotation of Three-Dimensional Objects. In: The Philosophy of Mind: Classical Problems/Contemporary Issues, 217.

[25] Miller, A., Sun, T., et al. (2013) Strategies for Training Technical and Non-Technical 
Surgical Skills. In: Proceedings of the Human Factors and Ergonomics Society Annual Meeting, Vol. 57, 658-662. https://doi.org/10.1177/1541931213571142

[26] Xu, X. and Harada, K. (2003) Automatic Surface Reconstruction with Alpha-Shape Method. The Visual Computer, 19, 431-443.

https://doi.org/10.1007/s00371-003-0207-1 